\title{
Das Dresdener Giesen-Urteil und die Auslegung des $\ 353$ b Abs. I StGB
}

\section{Zur Notwendigkeit der Aufgabe des Dogmas sog. »mittelbarer Gefährdungen«}

Der Freispruch des sächsischen Datenschutzbeauftragten Thomas Giesen vom Vorwurf der Verletzung des Dienstgeheimnisses gem. $\ 353 \mathrm{~b}$ StGB durch das LG Dresden am 7. I I. 200 I bezeichnet nicht nur das vorläufige Ende einer sächsischen cause celèbre. Gegenstand des Urteils sind an einem prominenten Fall strafrechtliche Grundfragen der Auslegung des $\ 353 \mathrm{~b} \mathrm{StGB}$, vor allem die umstrittene Figur sog. mittelbarer Gefährdung, die auf Revision der Staatsanwaltschaft auch den 5. (Leipziger) Senat des Bundesgerichtshofs beschäftigen werden.

\section{Das Urteil des LG Dresden ${ }^{\mathrm{I}}$}

\section{Sachverhalt}

Giesen hatte am 22. 8. 2000 zwei Vermerke des damaligen Justizministers Heitmann gegenüber Medienvertretern verlesen, wonach dieser I997 auf Bitte eines Parteifreundes, ihm den Stand eines Ermittlungsverfahrens gegen einen Dritten mitzuteilen, einen Abteilungsleiter bat, »auf beschleunigte Behandlung hinzuwirken « und ihm baldmöglichst zu berichten, und sodann die Unterrichtung des Parteifreundes vermerkte. Am 23. 8. 2000 sandte Giesen eine Datenschutzrechtliche Beanstandung dieser Vorgänge an seinen Petenten, den vormaligen Oberbürgermeister von Görlitz, und verteilte sie am gleichen Tag an Pressevertreter im Rahmen einer Pressekonferenz.

Der Vorgang führte zum Rücktritt des Ministers und zur Änderung des Organisationsstatuts für Staatsanwaltschaften hinsichtlich ihrer Berichtspflichten.

\section{Gründe}

Das Gericht sieht in den von Giesen weitergegebenen Informationen die Offenbarung von Gebeimnissen.

Dies sei indes nicht unbefugt und daher nicht rechtswidrig erfolgt. Zwar sei Giesen zur Amtsverschwiegenheit verpflichtet gewesen, und eine Regelung darüber, wann der Datenschutzbeauftragte sich an die Presse wenden dürfe, fehle im Sächsischen Datenschutzgesetz. Das Gericht hält indes die Voraussetzungen des $\ 34$ StGB für gegeben: Der ehemalige Justizminister habe in erheblicher Weise gegen das Sächsische Datenschutzgesetz und den Grundsatz der Gewaltenteilung verstoßen. In dieser Lage habe das Interesse, an die Öffentlichkeit zu gehen, das beeinträchtigte Interesse an der Wahrung von Geheimnissen überwogen; Giesen habe zuvor auf eine Stellungnahme des Ministers innerhalb einer von ihm gesetzten Frist gewartet und erst anschließend zur Verhinderung einseitiger Berichterstattung eine sachliche und vollständige Unterrichtung der Öffentlichkeit für erforderlich gehalten.

Das Gericht sieht zudem das Tatbestandsmerkmal der Gefährdung wichtiger öffent- 
licher Interessen nicht erfüllt. Weder das Ansehen der Justizverwaltung und der Justiz noch des Petenten noch des sächsischen Datenschutzbeauftragten sei gefährdet worden, vielmehr seien durch das Tun des Angeklagten interne Vorgänge aufgedeckt worden und Justiz und Datenschutzbehörde danach in den Augen der Öffentlichkeit gestärkt aus der »Affäre« hervorgegangen.

\section{Kritische Würdigung}

Den Freispruch vom Vorwurf des $\ 353$ b StGB stützt das Gericht somit auf zwei je für sich (»weder... - noch«) die Strafbarkeit ausschließende Gesichtspunkte; hierbei fällt auf, daß es die Rechtswidrigkeit vor der Tatbestandsmäßigkeit prüft, also zunächst die Rechtmäßigkeit im Einzelfall (rechtfertigender Notstand) begründet und erst danach (»es kommt hinzu«) die generelle Tatbestandsmäßigkeit (fehlende Gefährdung wichtiger öffentlicher Interessen) verneint. Der Zweck dieser Vorgehensweise bleibt unklar; denkbar ist, dass sich das Gericht im Rahmen der Notstandsprüfung eine umfassende Würdigung des Sachverhalts einschließlich der Bewertung der von Giesen gerügten ministeriellen Praktiken nicht nehmen lassen wollte, aber auch, dass es sein Ergebnis für die Revision doppelt absichern wollte, weil es eine andere Auslegung für jeden der genannten Gründe für möglich hielt.

Die Problematik der Prüffolge des Gerichts wirkt sich bei der Abwägung der widerstreitenden Interessen im Rahmen des $\ 34$ StGB aus, wonach das Interesse Giesens, als Leiter der Datenschutzbehörde an die Öffentlichkeit zu gehen, das beeinträchtigte Interesse an der Wahrung von Geheimnissen wesentlich überwogen habe. Wurden nämlich, wie das Gericht später ausführt, hierdurch wichtige öffentliche Interessen an der Geheimhaltung gar nicht gefährdet, so fehlt die Voraussetzung jeder Abwägung (weshalb ein »beeinträchtigtes« Interesse hier zunächst nur unterstellt werden kann).

Nachfolgend werden zunächst die Ausführungen des Gerichts zu den Tatbestandsmerkmalen des $\ 353$ b StGB diskutiert.

Geheimnis: Das Gericht sieht den Geheimnisbegriff des $\$ 353$ b StGB durch die genannten weitergegebenen Informationen erfüllt, da sie vor der Weitergabe durch Giesen erst einem begrenzten Personenkreis bekannt und daher noch nicht offenkundig waren. Das entspricht dem weiten Geheimnisbegriff der Rechtsprechung. ${ }^{2}$ Er ermöglicht bei dem Merkmal »Gefährdung wichtiger öffentlicher Interessen« die Einbeziehung mittelbarer Gefährdungen, wenn durch das Bekanntwerden der Offenbarung eines beliebigen Geheimnisses das Ansehen der Verwaltung in Gefahr gerät. ${ }^{3}$

Eben dies auszuschließen, ist das Anliegen von Lenckner und Perron; ${ }^{4}$ danach bedarf das Geheimnis bei $\mathbb{\int} 353$ b StGB einer teleologischen Reduktion auf solche Informationen, bei denen der geheimzuhaltende Sachverhalt ein »wichtiges öffentliches Interesse « betrifft. ${ }^{5}$ Dass ein Minister einem Parteifreund über den Stand eines Ermittlungsverfahrens berichtet, die Staatsanwaltschaft um dessen beschleunigte Behandlung gebeten und dass der Datenschutzbeauftragte dies als unzulässig beanstandet hat, ist sicher ein Sachverhalt, an dessen Geheimhaltung unmittelbar kein, erst recht kein wichtiges öffentliches Interesse besteht.

2 S. zuletzt $B G H, \mathrm{JZ}$ 2002, $4^{8}$ (49) m. w. N. .

3 Vgl. Lenckner/Perron, in: Schönke/Schröder, StGB, 26. Aufl. (200I), $\mathbb{3} 353$ b Rz. 6 f. m. w. N.

4 Ebd.; Perron, JZ 2002, 50 ff.

5 Lenckner/Perron (Fn. 3); Perron JZ 2002, 52: "Wenn eine Gefährdung wichtiger öffentlicher Interessen als unmittelbare Folge der Geheimnisoffenbarung eintritt, dann müssen diese wichtigen öffentlichen Interessen mit dem Gegenstand des Geheimnisses verbunden sein«. 
Umgekehrt wäre zu fragen, ob nicht ein öffentliches Interesse, nämlich ein solches der demokratischen Öffentlichkeit, an der Preisgabe der Information bestand.

$\mathrm{Da}$ sich erweisen wird, dass die Einbeziehung mittelbarer Gefährdungen in die Auslegung des $\ 353 \mathrm{~b} \mathrm{StGB}$ prinzipiell verfehlt ist, gibt es für einen weiten Geheimnisbegriff kein Bedürfnis. Perron ${ }^{6}$ hat jüngst die Kritik des 2. Senats des Bundesgerichtshofs an der von ihm und Lenckner vertretenen teleologischen Reduktion überzeugend zurückgewiesen. Der Freispruch hätte somit schon auf das Fehlen eines Geheimnisses gestützt werden können.

Gefährdung wichtiger öffentlicher Interessen: Der bemerkenswerte Kernsatz der Anklage lautet: »Bei Bekanntwerden der unter Ziffern I.-3. geschilderten Tatsachen war jeweils mit einem erheblichen Vertrauensverlust der Öffentlichkeit in die Tätigkeit des Sächsischen Staatsministers der Justiz sowie des Sächsischen Datenschutzbeauftragten zu rechnen. Dies nahm der Angeklagte jeweils zumindest billigend in Kauf «?

Damit ist die seit RG DStR I938, 32 I von der Rechtsprechung vertretene, I958 vom Bundesgerichtshof übernommene, ${ }^{8}$ von der ganz herrschenden Lehre hingegen abgelehnte ${ }^{9}$ sog. Theorie der mittelbaren Gefäbrdung angesprochen, wonach durch das (»drohende«) Bekanntwerden der Offenbarung eines beliebigen Geheimnisses als mittelbare Folge das allgemeine Ansehen der Verwaltung, das selbst ein wichtiges öffentliches Interesse darstelle, gefährdet werden könne.

Hier ging es bisher um zwei Fallgruppen:

- Am häufigsten sind die Fälle, in denen ein Amtsträger einem Dritte Informationen preisgegeben hatte, die nicht im öffentlichen Interesse, sondern zum Schutz eines Privaten geheim zu halten waren. ${ }^{\circ}$ Hier wurde eine mittelbare Gefährdung im Falle des (»drohenden«) Bekanntwerdens des Vorfalls in der Bevölkerung durch die dann zu erwartende oder eingetretene »Erschütterung « des Vertrauens in die Integrität von Behörden angenommen.

- Weiter ging es um Fälle, in denen nicht ein Privatgeheimnis, sondern ein innerdienstliches Geheimnis bekannt wurde (wie etwa durch Weitergabe einer Prüfungsaufgabe an Schüler durch einen Lehrer ${ }^{\mathrm{II}}$ ) und dadurch unmittelbar kein wichtiges öffentliches Interesse gefährdet wurde, aber ebenfalls ein Ansehensverlust der Behörde in der Öffentlichkeit eintreten konnte.

In diesen Fällen wurde somit nach Ablehnung einer unmittelbaren noch die Frage einer mittelbaren Gefährdung erörtert. ${ }^{\text {I2 }}$

Beiden Fallgruppen ist gemeinsam, dass die Tatsache des Geheimnisbruchs gleichsam aus Versehen nachträglich ans Licht der Öffentlichkeit gelangte.

Auch im vorliegenden Fall fehlt eine unmittelbare Gefährdung wichtiger öffentlicher Interessen. Seine Besonderheit besteht darin, dass ein Amtsträger sich gezielt über die Medien an die Öffentlichkeit wendet und diese so von seinem Bruch der Amtsverschwiegenheit und von den von ihm gerügten Vorgängen erfährt. Hier liegt eine Parallele zu dem bekannten Pätsch-Fall, ${ }^{\mathrm{1}}{ }^{\mathrm{I}}$ in dem es aber gerade um eine unmittelbare

$6 \mathrm{JZ} 2002,52$.

7 Urteil LG Dresden (Fn. I), S. 2.

8 BGHSt II, 40I/404; zuletzt bestätigt durch den 5. Senat des BGH in StV 2002, 26 (28): "Eine solche mittelbare Gefährdung kann nach der Rspr. des $B G H$ grundsätzlich ausreichen«.

9 Perron, JZ 2002, 52 Fn. 7 m. w. N.

I0 OLG Köln, GA I973, 57; OLG Düsseldorf, NStZ I985, I69 (170); OLG Köln, NJW ı988, 2489; LG Bad Kreuznach, CR I991, 37; LG Ulm, NJW 2000, 822; OLG Hamm, NJW 2000, I 278 (I 280); BGH, StV 2002, 26 (28).

I I $B G H S t$ I I, 40 I (404).

I 2 OLG Köln, NJW I989, I872 (1873).

I 3 BGHSt 20, 342; BVerfGE 28, I 94 ff. (s.u. 2). 
Gefährdung (der Zusammenarbeit des Bundesamtes für Verfassungsschutz mit alliierten Geheimdiensten) ging. ${ }^{14} \mathrm{Zu}$ erinnern ist daran, dass die Theorie der mittelbaren Gefährdung die mittelbaren Folgen der Tatsache des Gebeimnisbruchs ${ }^{15}$ in Gestalt des Ansehensverlustes der Behörde, der eintritt, wenn sich einer ihrer Beamten als unzuverlässig im Umgang mit Geheimnissen erweist, erfassen will.

Danach wäre vorliegend allein nach den Folgen des »Gangs an die Öffentlichkeit« durch den Behördenleiter Giesen für das Ansehen seiner Behörde zu fragen, während Anklage und Urteil auch den Ansehensverlust von Justizminister und Staatsanwaltschaft durch die von Giesen gegebenen Informationen thematisieren. Hierin ist eine weitere Erosion der Theorie der mittelbaren Gefährdung zu sehen.

In der Rechtsprechung der Oberlandesgerichte wird die Frage, wann bei mittelbaren Gefährdungen eine konkrete Gefahr vorliegt, nicht einheitlich beantwortet. Gegen den Standpunkt des OLG Köln, wonach das Vertrauen der Bevölkerung in die Verschwiegenheit von Behörden durch das Aufdecken und Bekanntwerden des Vorfalls erschüttert werden müsse (wofür Tatsachen wie Reaktionen der »seriösen «Presse und Proteste aus der Bevölkerung angegeben werden müssten ${ }^{16}$ ) wendet das BayObLG m. E. vom Standpunkt der mittelbaren Gefährdung konsequent - ein, wegen des Charakters des $\mathbb{S} 353 \mathrm{~b}$ als Gefährdungsdelikt sei die Vollendung vorverlegt; daher könne die Offenbarung gegenüber einem Einzelnen bereits genügen, wie etwa einem zur Veröffentlichung bereiten Journalisten. ${ }^{17}$ Danach hätte vorliegend bereits durch die Information der Medienvertreter Vollendung angenommen werden können, wenn das Gericht die Gefahr eines Ansehensverlustes der Behörden bejaht hätte. ${ }^{18}$

Einigkeit besteht unter den Anhängern der »mittelbaren Gefährdung «, dass aus dem »drohenden« oder eingetretenen Bekanntwerden des Geheimnisverrats nicht ohne weiteres auf eine Gefährdung wichtiger öffentlicher Interessen geschlossen werden kann, ${ }^{19}$ weil diesem Merkmal eine »Korrekturfunktion ${ }^{20}$ zukomme, weshalb auf die Besonderheiten des Einzelfalls abzustellen sei. Das BayObLG will insbesondere die Motivlage des Täters berücksichtigen, so dass, wer in einer Konfliktlage handle oder auf Missstände hinweisen wolle, eher Verständnis finde und das allgemeine Vertrauen weniger beeinträchtige als derjenige, der aus missbilligenswerten Gründen handle. ${ }^{21}$ Der 5. Senat des Bundesgerichtshofs zieht für die »hiernach gebotene Gesamtabwägung « Inhalt und Umfang der geheimhaltungsbedürftigen Daten, deren in Aussicht genommene Verwendung und die Person des Amtsträgers heran. ${ }^{22}$ Aus derartigen Kriterien werden dann von den Gerichten »quasi-demoskopische« oder normative Prognosen abgeleitet, ob bei Bekanntwerden des Geheimnisbruchs zu erwarten sei, dass das Vertrauen der Bevölkerung erschüttert und deshalb das Ansehen der Behörde geschädigt werde. ${ }^{23}$

I4 Im Arbeitsrecht werden parallele Fälle des »whistleblowing « im Zusammenhang mit Kündigungen diskutiert; s. Müller, NZA 2002, 42 I, der unter Fn. 26 auf BVerfGE 28, I9I verweist.

Is Schäfer, DStR 1938, 321: »Bekanntwerden nicht der offenbarten Tatsache, sondern der Tatsache der unbefugten Offenbarung «.

i6 NJW I988, 2489 (249I); vgl. (undeutlich) OLG Düsseldorf, NStZ I985, I70.

I7 BayObLG, NStZ-RR 1999, 299; vgl. Lenckner/Perron (Fn. 3), $\mathbb{3 5 3}$ b Rz. 6 a, wonach die von ihnen abgelehnte Rechtsprechung keineswegs folgerichtig verfahre.

I 8 Auf die Konsequenz, dass danach eigentlich die Pressekonferenz polizeirechtlich zur Verhütung von Straftaten hätte verboten werden können und - erkennt man mit dem BayObLG die Möglichkeit von Beihilfe in der anschließenden Phase bis zur materiellen Beendigung an - eine Strafverfolgung der veröffentlichenden Journalisten wegen Beihilfe möglich gewesen wäre (so explizit BayObLG [Fn. I7] m.krit.Anm. Behm, AfP 200I, 42 I (425)), sei hier nur hingewiesen.

I 9 BayObLG NStZ-RR I999, 300.

$20 B G H, \mathrm{StV} 2002,28$.

2 I BayObLG, NStZ-RR 1999, 299.

$22 B G H, \mathrm{StV} 2002,28$.

23 S. z. B. LG Ulm, NJW 2000, 822 (823); krit. Kublen, Nomos Kommentar zum StGB, $\$ 353$ b Rz. 25 f. 
Das LG Dresden geht hierüber hinaus: Es schließt eine Gefährdung des Ansehens von

Justizministerium, Justiz und Datenschutzbehörde mit folgenden Erwägungen aus: In der Öffentlichkeit sei beachtet worden, dass das Organisationsstatut für Staatsanwaltschaften neu gefasst wurde und schließlich ein Ministerwechsel eingetreten sei. Der »objektiven Öffentlichkeit« sei deutlich geworden, dass Staatsanwaltschaft und Gerichte auf die Einhaltung ihrer Unabhängigkeit bedacht seien. Das Ansehen des Datenschutzbeauftragten in der Öffentlichkeit sei gestärkt und nicht gefährdet worden. Und in der Öffentlichkeit sei der Eindruck entstanden, dass durch sein Tun verbotene Vorgänge und Verhaltensweisen aufgedeckt und die Justiz hierdurch gestärkt aus der Affäre hervorgegangen sei. ${ }^{24}$

Damit überspringt das Gericht die Phase einer möglichen Gefährdung durch Offenbaren an veröffentlichungsbereite Personen und die nächste Phase, in der der Vorfall durch die Veröffentlichung in der Bevölkerung bekannt wird, sowie deren unmittelbare Reaktion (die nicht mitgeteilt wird - Proteste, Leserbriefe? Diskussion im sächsischen Landtag ${ }^{25}$ ), die jedenfalls zunächst doch zu einer Schädigung des Ansehens der Behörden geführt haben kann, und betrachtet - sozusagen saldierend - die Folgen, indem es die erforderlichen Maßnahmen zur Wiederherstellung enttäuschten Vertrauens in die Reaktion der Öffentlichkeit mit einbezieht. ${ }^{26}$

So weit ist bisher noch kein Gericht gegangen - und dies aus gutem Grund: Dieser zeigt sich bei der Überlegung, dass die Konsequenz auch ganz anders hätte ausfallen können: Die mitgeteilten Verstöße wären von staatlicher Seite folgenlos geblieben, der Minister wäre nicht zurückgetreten, die Gefahr einer Wiederholung der Missstände wäre nicht ausgeräumt worden, und die Öffentlichkeit hätte eventuell mit ohnmächtigen Protesten und massiven Vertrauensverlusten reagiert - wäre das ein Grund gewesen, den Angeklagten, der diese oder jene Folge der von ihm veranlassten Veröffentlichung nicht vorhersehen konnte, aus $\mathbb{\$} 353 \mathrm{~b}$ StGB zu verurteilen? Der Rückschluss von nachtatbestandlichen Vorgängen bis zur Beendigung auf die fehlende Vollendung ist nicht zulässig, dies schon mangels objektiver Zurechenbarkeit, ohne dass es auf Vorsatz oder Fahrlässigkeit ankäme; und sähe man dies anders, nahm der Angeklagte sicher eine Beunruhigung der bisher uninformierten Öffentlichkeit in Kauf, um erst durch deren erhoffte Reaktion die Voraussetzungen zur Beseitigung der von ihm gerügten Missstände zu schaffen. In der Terminologie der »mittelbaren Gefährdung « war sein Vorsatz darauf gerichtet, bisher vorhandenes, aber unbegründetes Vertrauen ${ }^{27}$ zunächst zu erschüttern, was für den subjektiven Tatbestand an sich ausreicht.

Kritik der Theorie der mittelbaren Gefährdung: Die gravierenden strafrechtssystematischen Einwände, die Lenckner, ${ }^{28}$ Perron $^{29}$ und Kublen ${ }^{30}$ vorgetragen haben, sollen hier nicht nochmals wiederholt werden. ${ }^{31}$ Die Kritik fällt aber unter Einbeziehung teleologisch-verfassungsrechtlicher Gesichtspunkte weit grundsätzlicher aus:

Zunächst zeichnet sich schon das Gesetz nicht gerade durch musterhafte Tatbestandsbestimmtheit aus. So heißt es bereits in der amtlichen Begründung: »Die Frage, wann

24 LG Dresden (Fn. I), S. 9 f.

25 www.gewaltenteilung.de.debatte.

$26 L G$ Dresden (Fn. I). Das Ergebnis ist übrigens nur vorläufig, wenn die Öffentlichkeit wahrnimmt, dass der Urheber der Veröffentlichung eben deshalb angeklagt, zunächst freigesprochen, hiergegen Revision eingelegt wurde und erst das Urteil des Bundesgerichtshofs zeigen wird, ob das »Funktionieren des Systems « in Frage zu stellen ist.

27 So kritisch Schumann, NStZ i985, 170 (173).

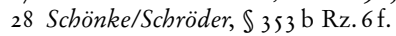

$29 \mathrm{JZ} \mathrm{2002,} \mathrm{50} \mathrm{(5I).}$

$30 \mathrm{NK} \int 353 \mathrm{~b} \mathrm{Rz.} 22 \mathrm{ff} ., 25 \mathrm{f}$.

3 I S. Behm, AfP 2000, 42 I (424). 
eine Gefährdung wichtiger öffentlicher Interessen vorliegt, kann oft zweifelhaft sein ${ }^{32}$ Hält man die Vorschrift dennoch auch unter Bestimmtheitsaspekten mit dem Bundesverfassungsgericht für mit dem Grundgesetz vereinbar, weil die Tatbestandsmerkmale durch eine langjährige Rechtsprechung hinreichend präzisiert seien, ${ }^{33}$ so gilt dies für mittelbare Gefährdungen in keiner Weise. Bedenklich ist schon der Hinweis, eine solche sei »immer anhand der Besonderheiten des Einzelfalles zu beurteilen «, ${ }^{34} »$ wobei die Voraussetzungen im einzelnen kaum geklärt sind «. ${ }^{35}$ Festzuhalten ist, dass es der Rechtsprechung in mehr als 60 Jahren nicht gelungen ist, die Unbestimmtheit durch Entwicklung tauglicher Kriterien zu überwinden. ${ }^{36}$ Versteht man Art. I03 Abs. 2 GG gleichsam als Verfassungsauftrag an die Judikatur, Strafgesetzen durch Auslegung schärfere Konturen und damit mehr Bestimmtheit zu verleihen, ${ }^{37}$ so verkehrt die Theorie der mittelbaren Gefährdung dies ins Gegenteil.

Hinsichtlich der Gefäbrdung wichtiger öffentlicher Interessen und Art. s Abs. I GG wurde oben (II 2 a) auf die Parallele des vorliegenden Falles zu dem Pätsch-Urteil des Bundesgerichtshofs hingewiesen; dies legt es nahe, die Maßstäbe des Bundesgerichtshofs hierfür fruchtbar zu machen, so weit die Parallele trägt: Seinerzeit ${ }^{38}$ hat der Bundesgerichtshof sogar für Staats- und (erst recht) für Amtsgeheimnisse aus $\ 353 \mathrm{~b}$ StGB ein Recht zur Rüge aus Art. s Abs. I GG abgeleitet und nach der Wechselwirkungstheorie danach unterschieden, ob aus der Sicht des Täters ein schwerer Verstoß gegen die verfassungsmäßige Ordnung vorliegt - dann darf unmittelbar die Öffentlichkeit angerufen werden, auch durch Amtsträger ${ }^{39}$ - oder ob ein weniger bedeutsamer Verstoß gerügt wird: Dann sei die Rechtswidrigkeit ausgeschlossen, wenn der Täter die Preisgabe auf das Notwendige beschränkt und zunächst i. S. einer »Stufentheorie« die Wege über die zuständigen Stellen und die Volksvertretung geht, bevor er die Öffentlichkeit anruft. ${ }^{4}$

Diese Lösung ist Ergebnis einer Interessenabwägung zwischen dem Recht zur Rüge von Missständen im öffentlichen Leben und der hierdurch erzwungenen Preisgabe von Staats- und Amtsgeheimnissen. ${ }^{4 \mathrm{I}}$ Sub specie $\ 353 \mathrm{~b}$ StGB ging es um eine unmittelbare Gefährdung wichtiger öffentlicher Interessen, nämlich der Zusammenarbeit mit den Abwehrdiensten der Alliierten. ${ }^{42}$

Im Dresdener Fall fehlt es, wie gezeigt, an einem solchen legitimen Gegeninteresse am Schutz von Geheimnissen, da an der Geheimhaltung der von Giesen mitgeteilten Vorgänge unmittelbar gerade kein öffentliches Interesse besteht. Nach den Maßstäben des Bundesgerichtshofs im Pätsch-Urteil hatte Giesen danach das Recht, sich an die Öffentlichkeit zu wenden. Diese Maßstäbe wurden vom Bundesverfassungsgericht gebilligt, das hervorhebt: "Die Aufmerksamkeit und das Verantwortungsbewusstsein des Staatsbürgers, der Missstände nicht nur zur Kenntnis nimmt, sondern sich auch für deren Abstellung einsetzt, ist eine wesentliche Voraussetzung für den Bestand der freiheitlichen demokratischen Ordnung (BVerfGE I 2, I I 3 [I 24 f]).

32 DJ 1936, 997.

33 BVerfGE 28, I9I (200); dieser Befund wirkt angesichts der bis 1970 geringen Zahl obergerichtlicher Entscheidungen wenig überzeugend.

34 BGH, StV 2002, 26 (28).

35 LG Ulm, NJW 2000, 822 (823).

$36 \mathrm{Behm}, \mathrm{StV}$ 2002, 29 (32); dort auch Kritik an der (immanent verdienstvollen) »tatbestandseinengenden Auslegung « und der »danach gebotenen Gesamtabwägung « durch $B G H, \mathrm{StV}$ 2002, 28.

37 Krey, Deutsches Strafrecht, AT Band I, Rz. I 2.

38 BGHSt 20, $342 \mathrm{ff}$.

39 Wobei offen gelassen wurde, ob hier Tatbestands- oder Rechtswidrigkeitsausschluss vorliegt.

40 BGHSt 20, 342, Leitsätze I-3; i. W. zust. Häberle, Das öffentliche Interesse (1970), S. 362 ff., der im erstgenannten Fall die Tatbestandslösung favorisiert (S. 365 , Anm. 63).

4I BGHSt 20, $362 \mathrm{f}$.

42 BGHSt 20, 38 I f. 
Das Grundrecht der freien Meinungsäußerung verdient, wenn es diesem Ziel dient,

besonderen Schutz; er kann auch dem Träger eines öffentlichen Amtes nicht versagt werden $\ll .{ }^{43}$

Sehen beide Gerichte im Fall nachvollziehbarer behördlicher Geheimhaltungsinteressen den Amtsträger zunächst im Rahmen der Abwägung zur Einhaltung des Dienstwegs verpflichtet (wobei Giesen als Behördenleiter sich bereits vergebens an Staatskanzlei und Minister gewandt hatte, so dass allenfalls noch die Anrufung des Landtags denkbar war), so ist, wo es nur um rechtswidrige Vorgänge in Behörden geht, deren Bekanntwerden unmittelbar die Behördentätigkeit nicht beeinträchtigt, die Wertung gerade umgekehrt vorzunehmen. Hier hat die demokratische Öffentlichkeit ein legitimes Interesse, von den Missständen unmittelbar zu erfahren, sich über deren Ausmaß eine Meinung zu bilden und öffentliche Kritik zu üben, die die Wiederherstellung rechtmäßiger Zustände erstrebt, begünstigt und vielfach erst ermöglicht.

Die Kritik, die Richard Schmid am Pätsch-Urteil geübt hat, ${ }^{44}$ wonach im Zweifel die Öffentlichkeit das erste Recht der Unterrichtung habe, traf das Richtige am falschen Objekt: Wurde nämlich mit der Zusammenarbeit mit alliierten Geheimdiensten unmittelbar ein öffentliches Interesse gefährdet, so stellte die Unterrichtung der Öffentlichkeit die denkbar intensivste Beeinträchtigung dieses legitimen Geheimhaltungsinteresses dar. ${ }^{45}$ Fehlt aber ein solches Interesse wie im vorliegenden Fall, so sind seine Ausführungen nach wie vor aktuell:

»In erster Linie hätte nämlich geprüft werden müssen, ob die Öffentlichkeit nicht ein begründetes Interesse und deshalb ein Recht darauf hatte, über die (realen oder unterstellten) Missbräuche unterrichtet $\mathrm{zu}$ werden, als Grundlage für die laufende demokratische Meinungs- und Willensbildung. (...) Lag aber ein öffentliches Interesse an Unterrichtung vor, so entsprach dem das Recht dessen, der die Fakten kannte, diese auch der Öffentlichkeit mitzuteilen, ein Recht, das jedem Bürger in gleicher Weise zusteht $\ll{ }^{46}$

Mit diesen verfassungsrechtlichen Wertungen ist die Theorie der mittelbaren Gefährdung unvereinbar. Entsprechendes gilt aber auch, wo ein Amtsträger Privatgeheimnisse oder weniger wichtige Amtsgeheimnisse unbefugt an Dritte weitergegeben hat, wenn dieser Vorfall öffentlich bekannt wird (oder die »Gefahr « des Bekanntwerdens mit entsprechenden Ansehens- und Vertrauensverlusten besteht). Wird hierin eine mittelbare Gefährdung wichtiger öffentlicher Interessen gesehen, so wird als strafrechtlich relevant nicht das eigentlich skandalöse Faktum der Preisgabe sensibler und deshalb rechtlich geschützter Informationen an Unbefugte gesehen (das nach $\ 203$ Abs. 2 StGB strafbar oder als Verstoß gegen die Verschwiegenheitspflicht Gegenstand eines Disziplinarverfahrens sein kann), sondern erst sein Bekanntwerden in der Öffentlichkeit. Mit anderen Worten wird hier ein »öffentliches Interesse«, dass die Öffentlichkeit vom Bruch der Amtsverschwiegenheit nichts erfährt, zum Schutzobjekt des $\ 353$ b StGB; nach derselben Logik kann dem Täter ein Strafbarkeitsvorwurf nicht gemacht werden, wenn der Vorfall in der Öffentlichkeit nicht bekannt wird, sie hierauf daher nicht kritisch reagieren kann und deshalb ihr Vertrauen in die Integrität der Behörden nicht erschüttert wird. ${ }^{47}$

Eine solche Strafbegründungslogik ist prinzipiell inakzeptabel. Hier wird in der höchstrichterlichen Rechtsprechung ein Dogma aus nationalsozialistischer Zeit 
(RG DStR 1938, 32I) tradiert, das gegen Geist und Buchstaben der Verfassung verstößt, weil es sich mit seiner Frontstellung gegen Meinungs-, Presse- und Informationsfreiheit und politische Willensbildung des Volkes gegen fundamentale Prinzipien der freiheitlich-demokratischen Ordnung wendet. Denn dadurch, dass die Theorie der mittelbaren Gefährdung erst das dem Täter zurechenbare »drohende« Bekanntwerden von Missständen pönalisiert, werden rechtswidrige Zustände in Behörden von öffentlicher Kritik abgeschottet. Damit werden demokratische Meinungsbildungsprozesse abgeschnitten, ohne die Missstände möglicherweise nicht abgestellt und die Verantwortlichen nicht zur Rechenschaft gezogen werden können. ${ }^{4}$ Mit öffentlichen Meinungsbildungsprozessen ist aber das, was in der Terminologie der mittelbaren Gefährdung so einseitig obrigkeitlich die »Erschütterung « des Vertrauens der Bevölkerung, die Gefährdung des Ansehens der Behörden genannt wird, zunächst unvermeidlich verbunden. Zu erinnern ist daran, dass es sich seinerzeit um das Vertrauen einer ihrer Grundrechte beraubten, von einer gelenkten Presse »ausgerichteten « Bevölkerung handelte, der jede »Öffentlichkeit « fehlte. Die fundamentale Bedeutung der »öffentlichen Meinung « für demokratische Willensbildung ${ }^{49}$ wird ins Gegenteil verkehrt. Hier verrät sich die Herkunft der »mittelbaren Gefährdung «: Sie ist untrennbares Element einer undemokratischen, autoritären Staats- und Gesellschaftsverfassung, die davon ausgeht, dass der zwischen Regierenden und Regierten bestehende Niveauunterschied eine freie Abgabe von Informationen nicht gestattet. ${ }^{\circ}$ Treffend formuliert Düwel: »Obwohl die staatstheoretischen Prämissen, von denen diese Praxis ihren Sinn empfing, längst entfallen sind, wirkt sich die Meinung, man dürfe >dem Volke nicht zu viel zumuten<, auch heute noch aus «. ${ }^{\text {I }}$

Schon 1979 artikulierte eine Minderheit im Rechtsausschuss des Bundestages entsprechende Kritik: Es werde der Begriff des öffentlichen Interesses »von der Rechtsprechung zu weit ausgelegt. Danach ist eine Gefährdung des öffentlichen Interesses auch dann gegeben, wenn das Vertrauen der Bevölkerung in die Unparteilichkeit der Verwaltung erschüttert werde. Eine solche Auslegung sei jedoch nicht mehr zeitgemäß. Denn der Bürger habe ein Anrecht darauf, Missstände in der Verwaltung zu erfahren ${ }^{52}$ Die vom 5 . Senat des Bundesgerichtshofs zu erhoffende Verabschiedung des Dogmas der mittelbaren Gefährdung wäre ein überfälliges Stück »Befreiung des Strafrechts vom nationalsozialistischen Denken ${ }^{53}$ Hierdurch würden auch keine Strafbarkeitslücken entstehen: Das Offenbaren von Privatgeheimnissen ist durch $\$ 203$ Abs. 2 StGB, von wichtigen Amtsgeheimnissen über die unmittelbare Gefährdung durch $\ 353 \mathrm{~b}$ StGB, von weniger wichtigen Amtsgeheimnissen durch disziplinarische Ahndung erfasst.

$\int 203$ Abs. 2 StGB: Da somit Giesens Gang an die Öffentlichkeit nicht zu einer Gefährdung wichtiger öffentlicher Interessen geführt haben kann, kam es insoweit auf die Frage der Rechtswidrigkeit nicht mehr an.

Auch der vorliegende Fall ist ein Beispiel dafür, dass Gerichte vielfach mittelbare Gefährdungen nach $\ 353 \mathrm{~b}$ StGB erörtern, wo in Wahrheit »nur « ein Delikt nach $\$ 203$ Abs. 2 StGB in Frage steht, aber ein Strafantrag nicht gestellt oder Verjährung eingetreten ist. ${ }^{54}$ Demgegenüber hat jüngst auch der Bundesgerichtshof auf die grund-

48 Vgl. Häberle (Fn. 40), S. 589 zum Fall Schwinkowski.

49 S. BVerfGE 20, $56 \mathrm{ff} ., 98 \mathrm{f}$.

s० Düwel, Das Amtsgeheimnis (1965) S. I79.

5 I Ebd.; dort (Anm. 20) Hinweis auf RG DStR I938, $32 \mathrm{I}$.

52 BT-Drs. 8/33I3, S. 6.

53 Wolf, JuS i 996, i 89 .

54 Vgl. OLG Düsseldorf, NJW I982, 2883 (2884); OLG Köln, NJW 1988, 2489; hierdurch wird aber kein effizienter Schutz von Privatgeheimnissen erreicht, da die konkrete Gefahr des Bekanntwerdens in der Öffentlichkeit vorliegen muss. 
sätzliche Strafbarkeit von Geheimnisverletzungen nach $\ 203$ Abs. 2 StGB im Rah-

men seiner »tatbestandseinengenden « Auslegung des $\ 353 \mathrm{~b}$ StGB hingewiesen."

Die von Giesen der Presse mitgeteilten Informationen über die Aktivitäten des Justizministers waren Geheimnisse i.S. d. $\ 203$ StGB, da sie vorher der Öffentlichkeit unbekannt waren. Unerheblich ist, auf welchen Lebensbereich sich das Geheimnis bezieht. Tatobjekt kann auch ein Geheimnis sein, das mit der politischen Betätigung des Geheimnisträgers verbunden ist, wobei der persönliche Lebensbereich auch das berufliche und das politische Wirken des Geheimnisträgers umfasst ${ }^{56}$. Gleichgültig ist hierbei, wie das Geheimhaltungsinteresse rechtlich zu bewerten ist. ${ }^{57}$ Dass bereits ein tendenziöser Zeitungsartikel erschienen war, ${ }^{8}$ ist für den Geheimnischarakter ohne Bedeutung, da es sich zunächst nicht um mehr als ein unbestätigtes Gerücht handelte. ${ }^{59}$

Da objektiver wie subjektiver Tatbestand gegeben waren, käme es nur auf die Rechtswidrigkeitsbewertung an. Hier hätten dann die im Ergebnis überzeugenden Ausführungen des LG Dresden zum rechtfertigenden Notstand ihren Ort. Ergänzend ist anzumerken: Wenn das Gericht die von Giesen gerügten ministeriellen Praktiken außer als schwerwiegenden Verstoß gegen die Regeln des Sächsischen Datenschutzgesetzes auch als gravierenden Eingriff seitens der Exekutive in die Judikative ansieht, so geht es um das Verhältnis des Justizministers zur Staatsanwaltschaft und deren staatsrechtliche Stellung. Diese wird zwar überwiegend zur Exekutive gerechnet, ${ }^{60}$ aber ihre starke Affinität zur Judikative betont, ${ }^{6 \mathrm{I}}$ weshalb sie ein relativ eigenständiges Organ der Rechtspflege sei, ${ }^{62}$ die nur den Rechtswillen, nicht den politischen Machtwillen des Staates zu vertreten habe. ${ }^{63}$ Weisungsberechtigte Stellen, wozu an der Spitze der Justizminister gehört, dürfen sich nicht von justizfremden Zwecken, von rechts- und sachwidrigen Erwägungen bei ihren Weisungen leiten lassen. ${ }^{64} \mathrm{Da}$ vorliegend der Justizminister offenbar aus parteipolitischen Gründen einem Parteifreund über ein Ermittlungsverfahren berichtete und auf dessen Beschleunigung drängte, ist der Bewertung dieses Vorgangs durch das Gericht zuzustimmen.

\section{Fazit}

Dass, wo ein Justizminister seine Kompetenzen zu parteipolitischen Zwecken überschreitet, der Datenschutzbeauftrage (dessen Amtes es ist, dem entgegenzutreten) deshalb angeklagt wird, weil er den Vorgang öffentlich macht, zeigt die Konsequenz einer prinzipiell inakzeptablen Auslegung des $\ 353$ b Abs. I StGB. Das LG Dresden hat Giesen im Ergebnis zu Recht freigesprochen. Es erscheint ausgeschlossen, dass der 5 . Senat des Bundesgerichtshofs dies anders sehen könnte. Nicht sicher ist, ob er bereit sein wird, sich mit der » mittelbaren Gefährdung « grundsätzlich auseinander zu setzen. Der vorliegende Fall bietet hierzu eine denkbar geeignete Gelegenheit, die so rasch und so deutlich kaum wiederkehren wird.

$55 B G H, \mathrm{StV}$ 2002, 26 (28).

56 Lenckner, in: Schönke/Schröder, StGB, $\$ 203$ Rz. 9 f.

57 Ebd., Rz. 7; dies ist eine Frage erst der Offenbarungsbefugnis.

58 LG Dresden (Fn. I), S. 5 .

59 Vgl. Lenckner (Fn. 3), Rz. 26; Lackner/Kühl, StGB, 24. Aufl. (200I) \203 Rz. I4.

60 Kleinknecht/Meyer-Goßner, StPO, 42. Aufl., vor \I4I GVG Rz.5; Volk, Strafprozessrecht, 2. Aufl. (200I) S. I9; anders Roxin, Strafverfahrensrecht, 25. Aufl. I998, S. 52: Weder der Exekutive noch der Judikative zuzurechnen, sondern selbstständiges Organ der Rechtspflege zwischen beiden.

6I Kleinknecht/Meyer-Goßner (Fn. 60); Institution sui generis, der dritten Gewalt zugeordnetes Organ der Rechtspflege, Mittlerin zwischen Exekutive und Gerichtsbarkeit.

62 Volk (Fn. 60); vgl. BVerfGE 9, 223 (228); 32, I 89 (216).

63 Kleinknecht/Meyer-Goßner (Fn. 60), \I46 GVG Rz. 5.

64 Ebd. 